\title{
Evaluation of the Safety and Efficacy of a Novel Home-Use Device with Diode Arrays and Contact Heating for Facial Skin Rejuvenation
}

\author{
J. Shaoul ${ }^{1}$, R. S. Mulholland ${ }^{2}$ \\ ${ }^{1}$ Laser \& Light Institute, Tel Aviv, Israel; ${ }^{2}$ SpaMedica, Toronto, Canada. \\ E-mail: mulhollandmd@spamedica.com
}

Received September $8^{\text {th }}$, 2011; revised October 28 ${ }^{\text {th }}$, 2011; accepted November $10^{\text {th }}, 2011$.

\begin{abstract}
Background: Phototherapy of photoaged skin has been attempted by lasers or intense pulsed light at the visible or near-infrared part of the spectrum. The use of red Light-Emitting Diodes (LED) delivering a low dose energy to the skin has been described for office and home use. Methods: In the present study, a portable home-use device is presented that combines fractional non-thermal red LED with mild contact heating. Forty subjects aged 30 - 55 presenting with facial lax skin, large pores, fine lines, wrinkles and brown pigmentation received 8 bi-weekly treatments for 4 weeks, using the Silk'n Reju/FaceFX device (Home Skinovations, Yokneam, Israel). Skin condition was evaluated before and 1, 2, and 3 months following the last treatment session. Grading of outcome was done according to an independent blinded evaluator and patient satisfaction. Results: Facial skin improvement of wrinkling and skin texture were apparent immediately and scored clinically after 3 months as excellent and considerable by $>90 \%$ of patients. Pigmentation improvement was less apparent. Overall facial skin improvement was noted by $77 \%$ of patients. Conclusions: The combination of fractional red LED and mild contact heating, using an at home portable device, proved to be safe and effective method to improve the quality of photoaged skin.
\end{abstract}

Keywords: LED, Home-Use, Photo-Aging, Anti-Aging

\section{Introduction}

Skin changes inflicted by chronological aging are intensified by environmental photoaging, which occurs as a result of prolonged exposure to UV radiation from the sun [1]. The most prominent changes are the appearance of uneven pigmentation and dark spots, as well as the reduced number of collagen and elastic fibers causing lax, sagging skin, fine wrinkles, and pronounced expression lines on the face [2].

Although skin aging cannot be prevented, some measures may help slow down and minimize the process. Ablative measures such as dermabrasion, excision, chemical peeling and laser resurfacing are effective, but with a high degree of adverse events and a long down time $[3,4]$. Non-ablative lasers and Intense Pulsed Light (IPL) technologies, based on selective photothermolysis [5] are more selective, but also presented burn hazards with consequent hyper pigmentation and scarring [6]. The nonablative, non-thermal modality of Light-Emitting Diodes
(LED) presents a mild but effective therapy of photoaged skin and has been successfully used by one or combination of diode wavelengths $[7,8]$.

The mechanism of action of low level LED is based on bio-stimulation [9]. At the red wavelength spectrum it has been shown to involve photomodulation of cell activity via dermal receptors that activate collagen synthesis along with inhibiting collagen degrading enzymes [10]. The wound healing process has also been reported to be stimulated by LED [11,12]. The mild and safe nature of the low dose LED treatment made it possible to use at home, after 8 bi-weekly sessions [13].

In the present study, contact heating has been added to fractional LED red light of the Silk'n Reju/FaceFX device in order to intensify the facial anti aging effect and have it visible after a short time. The aim of this study was to assess the safety and efficacy of a low level LED emission home-use device and to record the clinical outcome of facial skin rejuvenation following 8 bi-weekly treatments. 


\section{Materials and Methods}

\subsection{Device Specifications}

The Silk'n Reju/FaceFX home-use device (Home Skinovations, Yokneam, Israel) is a small, lightweight, portable, affordable LED system (Figure 1). It consists of a matrix of 24 LEDs in a spot size of $5 \mathrm{~cm}^{2}$, emitting at the red and infrared part of the light spectrum. The device has a heated metal plate with a built in skin surface gauge which ensures temperature cut-off at $41^{\circ} \mathrm{C}$. The motor is air-cooled.

\subsection{Study Participants}

Forty healthy patients, mostly females, with an age range of 30 - 55 years (mean age 41) were selected for two study sites in Laser \& Light Institute, Tel Aviv, Israel; and in SpaMedica, Toronto, ON, Canada.

Subjects were of various Fitzpatrick's skin types: II (16 subjects), III (14 subjects), and IV (10 subjects). All participants used the Silk'n Reju/FaceFX on their faces for treatment of at least one lesion type, as inclusion criteria. They were: facial fine lines and wrinkles with Fitzpatrick classification of wrinkling and elastosis class II and III [14], superficial melanin pigmentation or textural lesions of large pores, and sagging skin. Exclusion criteria included known sensitivity to laser treatment, any other cosmetic topical, injectable or oral treatment, as well as ablative and non-ablative laser/IPL/RF modality within the last 6 months. Informed consents to the treatment were obtained from all study participants prior to study commence.

\subsection{Treatment Technique and Protocol}

Patients were guided how to use the equipment and treated themselves under the guidance of a qualified person in the study sites clinics. Facial treatment zones were

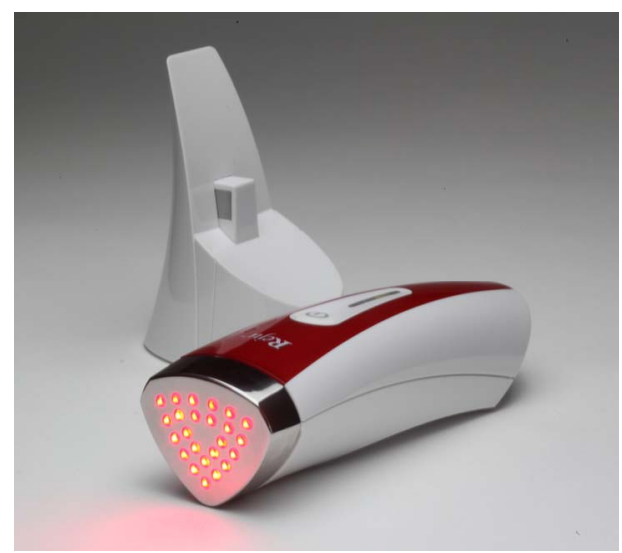

Figure 1. Silk'n Reju/FaceFX facial skin rejuvenation device and charging cradle. perioral and periorbital zones, as well as the cheeks and forehead. Each zone was treated in a constant circular move until the desired skin surface cut-off temperature was achieved, which took about 5 - 7 minutes per zone. Treatments were performed twice a week for 4 weeks (8 treatment sessions altogether).

\subsection{Clinical Assessments}

Patients were photographed before the first session to serve as a baseline, as well as 1, 2, and 3 months following the last session. Standardized front and profile closeup facial photos were taken. Scores were taken by blinded independent physician while comparing the photographs. Fine lines, wrinkles and skin laxity were graded in classes according to Fitzpatrick classification of wrinkling and elastosis from Class I (1-3), Class II (4-6), and Class III (7-9). Improvement from one class to a lower class or within the same class of wrinkling and elastosis were recorded as: none, poor, some, considerable and excellent response. Density and shade of the brown spots and pores size were evaluated according to the same scale by \% participants. For example, 94\% of participants experienced considerable improvement in wrinkling. Improvement was scored according to lesion: wrinkling comprising fine lines and wrinkles, melanin pigmentation, and overall akin texture comprising facial skin pores, elasticity and firmness. Patient satisfaction assessment was recorded for overall improvement of all facial zones and all lesion types according to the same scale.

\section{Results}

Results were visible immediately after a single application of the Silk'n Reju/FaceFX device, as shown in the split face treatment in Figure 2. Apart from transient erythema that resolved within 2 hours, improved skin

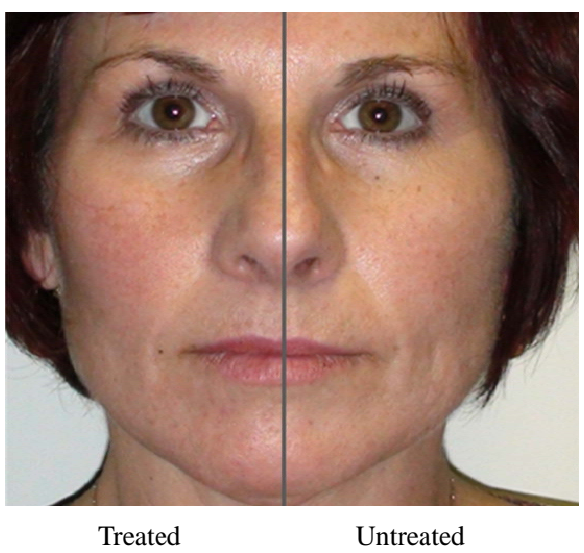

Figure 2. Split face showing immediate results (left) and 2 hours post treatment (right). 
texture, reduced periorbital lines and cheek bone lift are noticeable. However, records were taken at 3 follow-up points of 1, 2, and 3 month post last treatment. Perioral wrinkling (Figures 3 and 4), periorbital wrinkling (Figures 5-7), and forehead wrinkling (Figures 8 and 9) are presented and show marked improvement. Large pores (Figure 10), nasolabial folds (Figure 11), and pigment (Figure 12) treatments also showed softening of the appearance of lesions.

Results evaluated by a blind independent physician are summarized in Table 1. The results show that as time elapses, there is a gradual improvement in all lesions. At 3 months follow-up, $94 \%$ and $98 \%$ of patients were evaluated as having excellent or considerable improvement in wrinkling and skin texture, respectively. Improvement of pigmentation was less apparent and was recorded after 3 months as $45 \%$ of patients having excellent or considerable improvement. There were no non-responders, and $5 \%$ of patients showed poor results only for pigmentation.

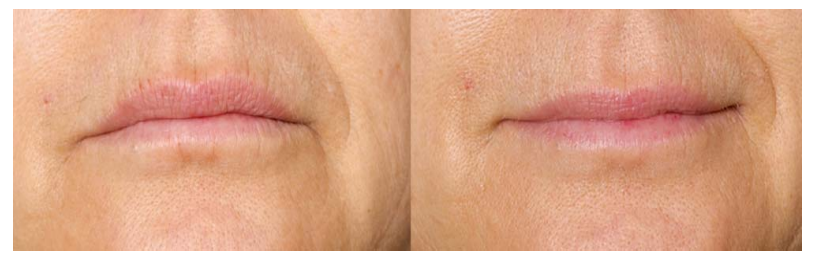

Figure 3. Upper lip smoker lines before (left) and after 1 month (right).

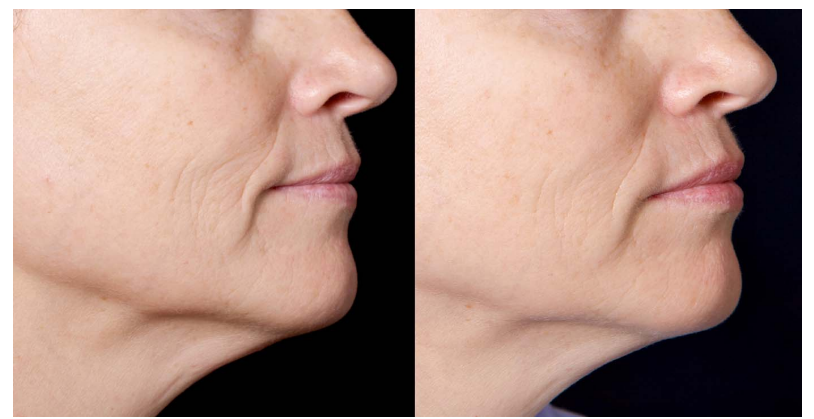

Figure 4. Perioral wrinkling before (left) and after 1 month (right).

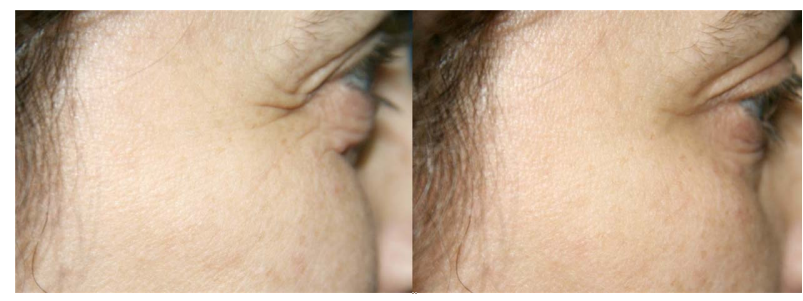

Figure 5. Female periorbital wrinkling (crow's feet) before (left) and after 3 months.

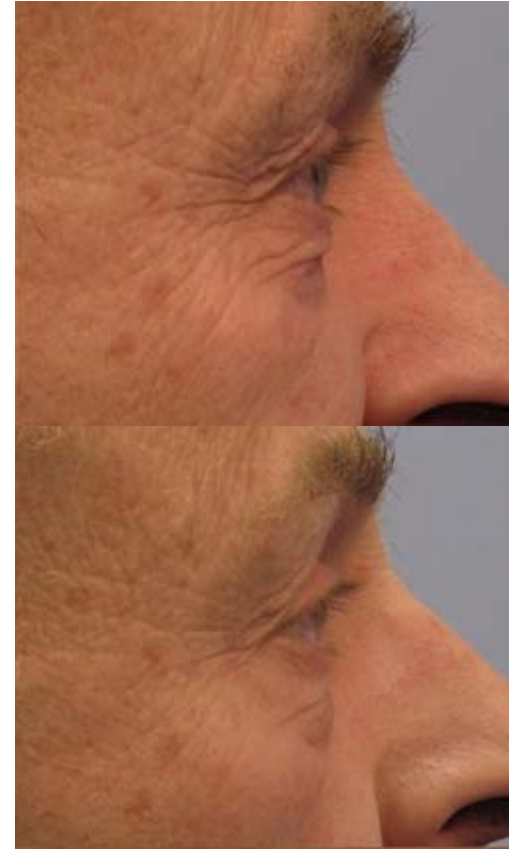

Figure 6. Male periorbital wrinkling (crow's feet) before (left) and after 3 months.

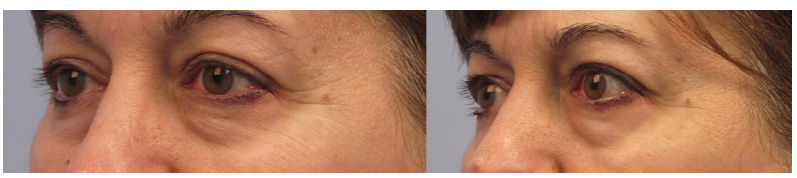

Figure 7. Female periorbital wrinkling under the eyes before (left) and after 2 months.

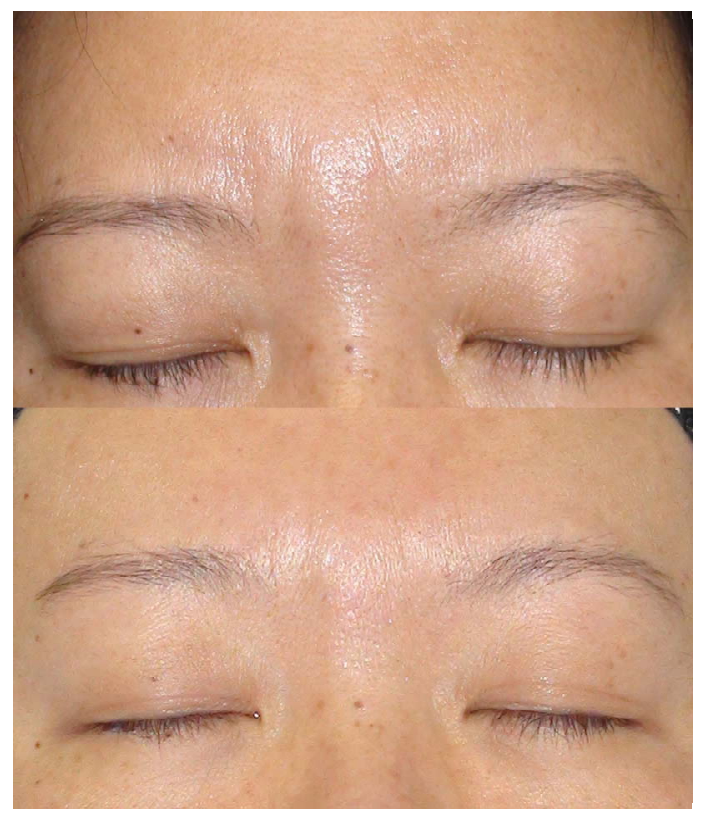

Figure 8. Forehead wrinkling before (top) and after 2 months (bottom). 


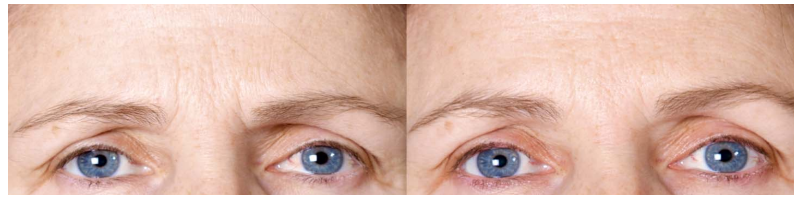

Figure 9. Forehead wrinkling before (left) and after 1 month.

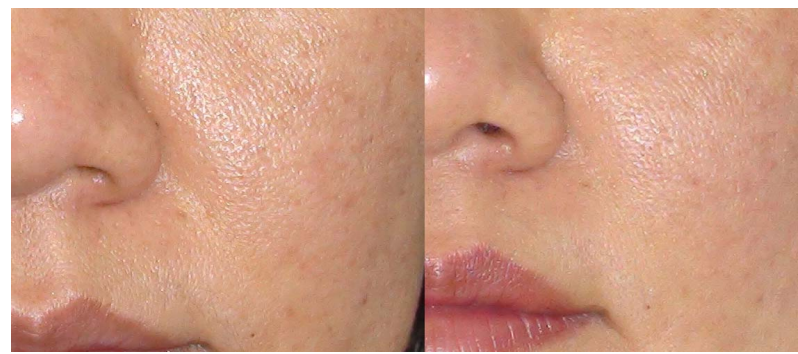

Figure 10. Large pores before (left) and after 2 months.

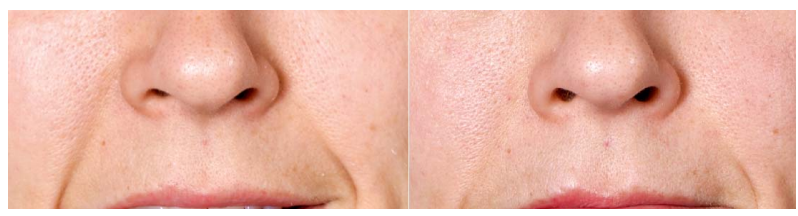

Figure 11. Nasolabial folds before (left) and after 1 month.

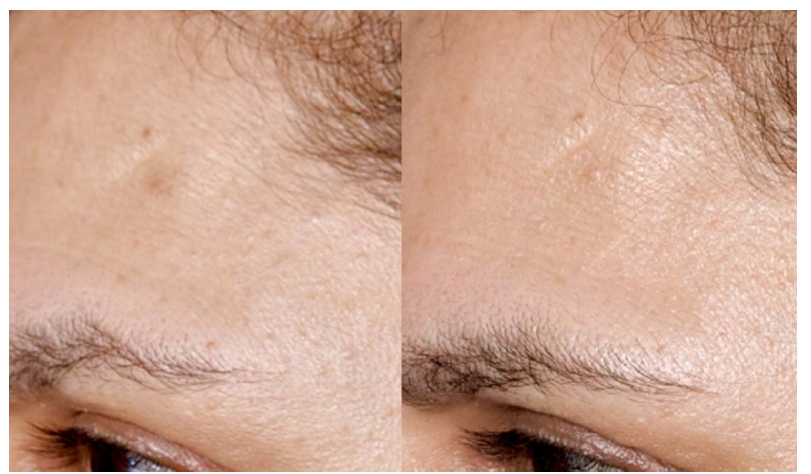

Figure 12. Forehead pigmentation before (left) and after 1 month.

Most patients expressed their degree of satisfaction to the overall improvement of facial skin after 1,2 , and 3 month follow-up sessions, as described in Table 2. Patients related to skin texture (pores, elasticity and firmness), to fine line and wrinkles and to pigmentation. No one experienced lack of response. Most patients (88\% $91 \%$ ) reported considerable and some response at all 3 points of follow-up.

Slight erythema and edema was noted in most patients within 5 minutes and lasted for about 30 minutes post treatment. The treatment was very comfortable for all patients who experienced pleasant warmth without any burning sensation. No complications were recorded through the study.

\section{Discussion}

This study has proven that the Silk'n Reju/FaceFX homeuse device is clinically safe and effective in improving the appearance and quality of aged and photo-aged facial skin. The objective clinical assessment by a blind independent physician usually displayed higher scores than the subjective patient satisfaction scores. Thus, after 3 months, the highest response of wrinkling and skin texture was scored as $23 \%$ and $25 \%$, respectively by a physician, whereas only $12 \%$ of patients scored excellent overall response. Considerable response was objectively evaluated as $71 \%$ - 73\% and subjectively as $65 \%$. The fact that the overall improvement scored by the patients included also the pigmentation that presented the least dramatic results may explain the difference.

The lower improvement scores of pigmentation may be explained by the mild heat that is effective for biostimulation, but not sufficient for complete melanin coagulation. Pigments that are deeper than the epidermis will not respond either.

Increasing gradual improvement with time was noted by both scores, which is in accordance with new collagen synthesis schedule. Improvement in photoaging is visible as early as 1 month post treatment and is becoming more apparent after 2 and 3 months. In fact immediate results can also be seen, but even 1 month follow-up is early in comparison to LED based devices [13,15]. The intensifying effect of earlier results is very likely due to the additional heating.

The overall efficacy of the device may be explained by its dual use of energies-LED and contact heating. The low dose optical energy of the non-thermal LED may contribute to bio-stimulation of the dermal fibroblasts, thus increasing the mass of new collagen and elastic fibers concomitantly with inhibiting the degrading enzymes down to the deep dermis $[9,10]$. This is manifested as improved wrinkling (fine lines and wrinkles) and skin texture (skin pores, elasticity and firmness).The optical energy of the LED along with the superficial penetration of heat radiation may heat the epidermis and render the melanin brown spots more fragile. In addition, the contact heating may also help in collagen contraction.

Safety of the Silk'n Reju/FaceFX device has been demonstrated through lack of complications and patient comfort during treatment. It may be due to the low energy of the LED and to the safety limit of cut-off temperature of the contact heating.

\section{Conclusions}

The application of a home-use fractional LED device for 
Evaluation of the Safety and Efficacy of a Novel Home-Use Device with Diode Arrays and

Contact Heating for Facial Skin Rejuvenation

Table 1. Physician evaluation of response of various facial lesions at 3 follow-up points.

\begin{tabular}{|c|c|c|c|c|c|c|c|}
\hline $\mathbf{N}$ & Lesion & $\begin{array}{l}\text { Follow-up } \\
\text { Month }\end{array}$ & $\begin{array}{c}\text { Excellent } \\
\text { Response \% }\end{array}$ & $\begin{array}{c}\text { Considerable } \\
\text { Response \% }\end{array}$ & $\begin{array}{c}\text { Some } \\
\text { Response \% }\end{array}$ & $\begin{array}{c}\text { Poor } \\
\text { Response \% }\end{array}$ & $\begin{array}{c}\text { No } \\
\text { Response \% }\end{array}$ \\
\hline \multirow{3}{*}{36} & \multirow{3}{*}{ Wrinkling } & 1 & 5 & 23 & 72 & 0 & 0 \\
\hline & & 2 & 15 & 60 & 25 & 0 & 0 \\
\hline & & 3 & 23 & 71 & 6 & 0 & 0 \\
\hline \multirow{3}{*}{18} & \multirow{3}{*}{ Pigmentation } & 1 & 0 & 30 & 63 & 7 & 0 \\
\hline & & 2 & 1 & 38 & 56 & 7 & 0 \\
\hline & & 3 & 3 & 42 & 50 & 5 & 0 \\
\hline \multirow{3}{*}{35} & \multirow{3}{*}{ Skin Texture } & 1 & 10 & 66 & 34 & 0 & 0 \\
\hline & & 2 & 21 & 57 & 22 & 0 & 0 \\
\hline & & 3 & 25 & 73 & 2 & 0 & 0 \\
\hline
\end{tabular}

Table 2. Overall patient satisfaction at $\mathbf{3}$ follow-up points.

\begin{tabular}{ccccccc}
\hline N & $\begin{array}{c}\text { Follow-up } \\
\text { Month }\end{array}$ & $\begin{array}{c}\text { Excellent } \\
\text { Response \% }\end{array}$ & $\begin{array}{c}\text { Considerable } \\
\text { Response \% }\end{array}$ & $\begin{array}{c}\text { Some } \\
\text { Response \% }\end{array}$ & $\begin{array}{c}\text { Poor } \\
\text { Response \% }\end{array}$ & $\begin{array}{c}\text { No } \\
\text { Response \% }\end{array}$ \\
\hline 40 & 1 & 2 & 49 & 42 & 7 & 0 \\
39 & 2 & 4 & 54 & 33 & 23 & 0 \\
38 & 3 & 12 & 65 & 23 & 0 \\
\hline
\end{tabular}

facial skin rejuvenation of photoaged skin has been demonstrated in this study. The safe and effective treatment makes it a suitable and valuable modality for home use.

\section{REFERENCES}

[1] Y. Takema, Y. Yorimoto, M. Kawai and G. Imokawa, "Age-Related Changes in the Elastic Properties and Thickness of Human Facial Skin,” British Journal of Dermatology, Vol. 131, No. 5, 1994, pp. 641-648. doi:10.1111/j.1365-2133.1994.tb04975.x

[2] A. M. Kligman, "Photoaging," In: T. B. Fitzpatrick, A. Z. Eisen, K. Wolf, I. M. Freedberg and K. F. Austen, Eds., Dermatology in General Medicine, 3rd Edition, MacGraw-Hill, New York, 1986, pp. 1470-1475.

[3] D. M. Duffy, "Informed Consent for Chemical Peels and Dermabrasion,” Dermatologic Clinics, Vol. 7, No. 1, 1989, pp. 183-185.

[4] O. Shasaldeen, J. D. Peterson and M. P. Goldman, "The Adverse Events of Deep Fractional $\mathrm{CO}_{2}$," Lasers in Surgery and Medicine, Vol. 43, No. 6, 2011, pp. 453-456. doi:10.1002/lsm.21079

[5] R. R. Anderson and J. A. Oarish, "Selective Photothermolysis: Precise Microsurgery by Selective Absorption of Pulsed Radiation,” Science, Vol. 220, No. 4596, 1983, pp. 524-527. doi:10.1126/science.6836297

[6] C. A. Nanni and T. S. Alstaer, "Complications of Cutaneous Laser Surgery,” Dermatologic Surgery, Vol. 24,
No. 2, 1998, pp. 209-219. doi:10.1016/S1076-0512(97)00286-0

[7] J. W. Kim, “Clinical Trial of Nonthermal 633 nm Omnilux LED Array for Renewal of Photoaging: Clinical Surface Profilometric Results,” Korean Society of Lasers in Medicine and Surgery, Vol. 9, No. 1, 2005, pp. 69-76.

[8] B. Russell, L. R. Reilly and N. A. Kellet, "A Study to Determine the Efficacy of Combination LED Light Therapy (633 nm and $830 \mathrm{~nm}$ ) in Facial Skin Rejuvenation," Journal of Cosmetic Laser Therapy, Vol. 7, No. 3-4, 2005, pp. 196-200. doi:10.1080/14764170500370059

[9] D. Barolet, "Light-Emitting Diodes (LEDs) in Dermatology,” Seminars of Cutaneous Medicine and Surgery, Vol. 27, No. 4, 2008, pp. 227-238. doi:10.1016/j.sder.2008.08.003

[10] R. A. Weiss, D. H. Macdaniel, R. G. Geronemus and M. A. Weiss, "Clinical Trial of a Novel Non-Thermal LED Array for Reversal of Photoaging," Lasers in Surgery and Medicine, Vol. 36, No. 2, 2005, pp. 85-91. doi:10.1002/lsm.20107

[11] K. C. Smith, "The Photobiological Basis of Low-Level Laser Radiation Therapy,” Laser Therapy, Vol. 3, 1991, pp. 19-24.

[12] A. P. de Sousa, A. D. de Aguiar Valença Neto, A. M. Marchionni, M. de Araújo Ramos, J. A. Dos Reis Júnior, M. C. Pereira, M. C. Cangussú, S. R. de Almeida Reis and A. L. Pinheiro, "Effect of LED Phototherapy ( $\lambda 700 \pm$ $20 \mathrm{~nm}$ ) on TGF- $\beta$ Expression During Wound Healing: An 
Immunohistochemical Study in a Rodent Model," Photomedical and Laser Surgery, Vol. 29, No. 9, 2011, pp. 605-601.

[13] N. S. Sadick, "A Study to Determine the Efficacy of a Novel Handheld Light-Emitting Diode Device in the Treatment of Photoaged Skin,” Journal of Cosmetic Dermatology, Vol. 7, 2008, pp. 263-267. doi:10.1111/j.1473-2165.2008.00404.X

[14] M. H. Gold, M. P. Goldman, J. Rao, A. S. Carcamo and M. Ehrlich, "Treatment of Wrinkles and Elastosis UsingVacuum-Assisted Bipolar Radiofrequency Heating of the
Dermis,” Dermatologic Surgery, Vol. 33, No. 3, 2007, pp. 300-309. doi:10.1111/j.1524-4725.2007.33064.X

[15] S. Y. Lee, K. H. Park, J. W. Choi, J. K. Kwon, D. R. Lee, M. S. Shin, J. S. Lee, C. E. You and M. Y. Park, “A Prospective, Randomized, Placebo-Controlled, Double Blinded and Split-Face Clinical Study on LED Phototherapy for Skin Rejuvenation: Clinical, Profilometric, Histologic, Ultrastructural and Biochemical Evaluations and Comparison of Three Different Treatment Settings," Journal of Photochemistry and Photobiology, Vol. 88, No. 1, 2007, pp. 51-67. doi:10.1016/j.jphotobiol.2007.04.008 